\title{
DNA topoisomerase II enzyme activity appears in mouse sperm heads after fertilization
}

\author{
A. S. Elsheikh*, Y. Takahashi and H. Kanagawa \\ Laboratory of Theriogenology, Department of Veterinary Clinical Sciences, Faculty of Veterinary Medicine, Hokkaido \\ University, Sapporo 060 - 0818, Japan. \\ Accepted 24 April, 2010
}

\begin{abstract}
Sperm suspensions of 4 male mice (A, B, C and D), having an initial motility grade of 3.5 were used to examine the presence of DNA topoisomerase II (top 2) activity in sperm heads. The initial percentage motile of male A was $75 \%$, male B was $80 \%$, male C was $70 \%$ and male D was $60 \%$. Top 2 activity was examined by testing the effect of etoposide (ETO), a specific top 2 blocker, on sperm motility, fertilizing ability and formation of the male pronuclei. Sperm suspension drops $(0.5 \mathrm{ml})$ and fertilization drops $(0.4$ $\mathrm{ml}$ ) were made from TYH medium with $50 \mu \mathrm{g} / \mathrm{ml}$ ETO (treatments) or TYH without ETO (controls). Sperm suspensions were made from epididymal sperms of the above males in treatments and controls and incubated for $3 \mathrm{~h}$. Mature mouse oocytes $(n=461)$ were co-cultured with capacitated sperms in the treatments fertilization drops for $5 \mathrm{~h}$. Other oocytes $(n=437)$ were co-cultured with capacitated sperms in the controls fertilization drops. The oocytes were further cultured for $24 \mathrm{~h}$ in KSOM with ETO (for treatments) and KSOM without ETO (for controls). Six sperm motility indexes (SMI) for each male were recorded at $30 \mathrm{~min}$ interval according to the formula, $\mathrm{SMI}=(\text { grade })^{2} \times \%$ motile. The fertilization rates and nuclear events were assessed by observing the pronuclei under an inverted microscope and finding the sperm heads in whole mounts from the oocytes that failed to make pronuclei. The SMI of the treatments and the controls increased gradually and reached peak values after $2 \mathrm{~h}$ of incubation. No differences $(p>0.05)$ were observed among SMI of the treatments and the controls. However, treatment reduced $(p<0.05)$ the fertilization rate and completely inhibited the formation of the pronuclei. All the oocytes fertilized in treatments $(n=412)$ failed to form pronuclei and all had enlarged or decondensed sperm heads, whereas $92.6 \%$ of the oocytes fertilized in the controls $(n=378)$ had pronuclei and only 30 $(7.4 \%)$ oocytes had enlarged or decondensed sperm heads. Neither sperm motility nor fertilization were inhibited with ETO. However, the formation of the pronuclei was blocked. It was concluded that ETO has no effect on sperm capacitation and top 2 activity appears in mouse sperm heads after oocyte penetration.
\end{abstract}

Key words: DNA topoisomerase II, sperm, treatment, mice.

\section{INTRODUCTION}

DNA topoisomerase II enzyme (top 2) is a structural component of the metaphase chromosomes (Earnshow

*Corresponding author. E-mail: adilelgarrai@yahoo.com. Current address: Department of Reproduction and Obstetrics, Faculty of Veterinary Medicine, University of Khartoum, Shambat P.O. Box 32, Sudan.

Abbreviations: ETO, Etoposide; top 2, DNA topoisomerase II enzyme; ICR, imprinting control region; fpm, forward progressive movement; i.p, intraperitoneal injection. et al., 1985a, b; Gasser et al., 1986). This enzyme is essential for chromosome separation, condensation and decondensation during mitosis (Dinardo et al., 1994; Wright and Schatten, 1990; Downes et al., 1991). In sperm heads, the DNA is condensed and there are no clear chromosomes, thus, the activity of top 2 was not found in frog sperms (Gaudio and Risely, 1984) and rat sperms (Chen and Longo, 1986).

Supplementation of etoposide (ETO) to the fertilization medium of mouse oocytes did not inhibit oocyte fertilization but it inhibited exit from meiotic arrest and the formation of male and female pronuclei (Elsheikh et al., 
2004). However, the fertilization rate was low. In our previous work (Elsheikh et al., 2004), the reduction of fertilization rate was attributed to the effect of ETO on cumulus cells surrounding the oocytes, but a probable effect of ETO on the sperm motility and capacitation was also expected.

If ETO is supplemented to the sperm suspension and/or fertilization drops, it might inhibits capacitation and/or fertilization. After sperm penetration, the condensed DNA will undergo decondensation to form male pronuclei, and top 2 is expected to appear in the male pronucleous. If the fertilization is carried in a medium supplemented with ETO, the formation of the male pronuclei will be inhibited.

Therefore, in the current investigation the influences of ETO supplementation, to sperm suspension and fertilization drops, on mouse sperm motility, capacitation, fertilizing ability and the formation of the male pronuclei were investigated.

\section{MATERIALS AND METHODS}

\section{Preparation of culture media}

Sperm suspension and fertilization drops were prepared from TYH medium (Toyoda et al., 1971). The medium was supplemented with $4 \mathrm{mg} / \mathrm{ml}$ bovine serum albumin (BSA; Sigma, Chemical Co., U.S.A) and at time of use, it was supplemented with $50 \mu \mathrm{g} / \mathrm{ml}$ ETO (Sigma) as described by Elsheikh et al. (2004). Control sperm suspension and fertilization drops were made from ETO-Free TYH. The media were filtered by a filter sterilizer (Gellman Science, Michigan, U.S.A). Sperm suspension drops $(0.5 \mathrm{ml})$ and fertilization drops $(0.4$ $\mathrm{ml}$ ) were made, from ETO supplemented (treatments) and ETOFree TYH (controls), in the center of sterilized tissue culture dishes (35 $\times 10 \mathrm{~mm}$, Nunc, Roskilde, Denmark). Thirty microlitres drops of KSOM medium (Erbach et al., 1994) supplemented with ETO were prepared on tissue culture dishes (Nunc), for further culturing, the oocytes were co-cultured with sperms in the treatments. Thirty microlitres drops of KSOM without ETO were prepared on tissue culture dishes (Nunc) ; for further culturing, the oocytes were cocultured with sperms in the controls. The drops were covered with paraffin oil and kept over night in $\mathrm{Co}_{2}$ incubator at $37^{\circ} \mathrm{C}$ under $5 \%$ $\mathrm{CO}_{2}$ in air until used.

\section{Preparation of sperm suspensions}

Spermatozoa were obtained from the caudae epididymides of 10 male imprinting control region (ICR) mice of proven fertility as described by Toyoda and Chang, (1974). The male mice were sacrificed by cervical dislocation and their caudae epididymides were removed aseptically and placed into the paraffin oil beside the treatments and controls suspension drops. They were punctured with 25 gauge needle and 2 - 3 drops of the dense sperm mass were introduced into the suspension drops. Thereafter, the suspension drops dishes were transferred to the incubator.

\section{Evaluation of sperm motility}

The sperm motility was assed using the sperm motility index (SMI) described by Bavister (1974) and Bavister and Andrews (1988). According to this SMI, two parameters of sperm motility were subjectively evaluated. These parameters were sperm motility grade and percentage motile. There were 6 grades of sperm motility according to this evaluation; $0=$ no movement; $1.0=$ twisting no forward progressive movement (fpm); $2.0=$ slow fpm; 3.0 = good purposeful fpm; $4.0=$ rapid fpm without hyperactivation; 5.0 = hyperactivation (capacitated sperm). The percentages of motile sperms were evaluated under a light microscope at a low magnification of $\times 20$. The SMI were calculated by combing the percentage motile of each sample with its sperm motility grade according to the formula, $\mathrm{SMI}=(\text { grade })^{2} \times \%$ motile. The SMI were counted as in Table 1 which was designed by Bavister (1974).

\section{Oocytes collection}

Six-week old ICR female mice were superovulated by intraperitoneal injection (i.p) injection of 5 I.U of eCG (Serotropin, Teikoku Zoki, Tokyo, Japan). Forty-eight hours later they were i.p injected with 5 I.U of hCG (Gonatropin, Teikoku Zoki). Fourteen hours later they were sacrificed and their oviducts excited and placed into the paraffin oil beside the fertilization drops (treatments and controls). The ampullae of the oviducts were torn with 25 gauge needle and the oocyte cumulus complexes and introduced into the fertilization drops. The fertilization drops were then returned to the incubator and kept until inseminated with capacitized sperms.

\section{Gamete co-culture}

After incubating sperm suspensions for $2 \mathrm{~h}$ the sperms are considered capacitized (Toyoda et al., 1971). From sperm suspensions having sperm concentration of $1-3 \times 10^{7}$ spermatozoa $\mathrm{ml}, 20 \mu \mathrm{l}$ were introduced into the fertilization drops of treatments and controls. The final sperm concentration in the fertilization drops was $0.4-1.4 \times 10^{6} \mathrm{spermatozoa} / \mathrm{ml}$. The gamete co-culture was allowed to continue for $5 \mathrm{~h}$.

\section{Culture of fertilized oocytes and assessment of nuclear events}

The oocytes co-cultured in treatments and controls fertilization drops were further cultured in KSOM prepared for treatments and KSOM prepared for controls, respectively. The culture was allowed to continue for $24 \mathrm{~h}$. The pronuclear formation was assed under an inverted microscope at $\times 20-\times 40$. The oocytes that failed to form pronuclei were whole mounted and fixed in acetoethanol and stained with acetoorcien and examined under a light microscope to evaluate nuclear events.

\section{Experimental design}

\section{Experiment I}

This experiment is a two factorial design to test the effects of time of incubation and ETO supplementation to the sperm suspension drops on sperm motility and sperm capacitation. Four treatments and 4 control sperm suspensions having 3.5 after $30 \mathrm{~min}$ of incubation were selected from 4 male mice ( $A, B, C$, and $D)$. The initial percentage motile of sperm suspensions from male $A=75 \%$, male $B=80 \%$, male $C=60 \%$ and male $D=70 \%$. Six SMI for treatments and controls were recorded at $30 \mathrm{~min}$ interval and the assessment of SMI continued for $3 \mathrm{~h}$. The SMI were compared among the treatments and the controls.

\section{Experiment II}

This is a simple factorial design to test the effects of ETO supple- 
Table 1. Numerical values of sperm motility indexes ${ }^{\mathbf{a}}$.

\begin{tabular}{|c|c|c|c|c|c|c|c|c|}
\hline \multirow{2}{*}{$\begin{array}{c}\text { Percentage } \\
\text { motile }\end{array}$} & \multicolumn{8}{|c|}{ Motility grade $^{b}$} \\
\hline & 1.5 & 2 & 2.5 & 3 & 3.5 & 4 & 4.5 & 5 \\
\hline 5 & 11 & 20 & 31 & 45 & 61 & 80 & 101 & 125 \\
\hline 10 & 23 & 40 & 63 & 90 & 123 & 160 & 203 & 250 \\
\hline 20 & 45 & 80 & 125 & 180 & 245 & 320 & 405 & 500 \\
\hline 25 & 56 & 100 & 156 & 225 & 306 & 400 & 506 & 625 \\
\hline 30 & 68 & 120 & 188 & 270 & 368 & 480 & 608 & 750 \\
\hline 35 & 79 & 140 & 219 & 315 & 429 & 560 & 709 & 875 \\
\hline 40 & 90 & 160 & 250 & 360 & 490 & 640 & 810 & 1000 \\
\hline 45 & 101 & 180 & 281 & 405 & 551 & 720 & 911 & 1125 \\
\hline 50 & 113 & 200 & 313 & 450 & 613 & 800 & 1013 & 1250 \\
\hline 55 & 124 & 220 & 344 & 495 & 674 & 880 & 1114 & 1375 \\
\hline 60 & 135 & 240 & 375 & 540 & 735 & 960 & 1215 & 1500 \\
\hline 65 & 146 & 260 & 406 & 585 & 795 & 1040 & 1316 & 1625 \\
\hline 70 & 158 & 280 & 438 & 630 & 858 & 1120 & 1418 & 1750 \\
\hline 75 & 164 & 300 & 469 & 675 & 919 & 1200 & 1519 & 1875 \\
\hline 80 & 180 & 320 & 500 & 720 & 980 & 1280 & 1020 & 2000 \\
\hline 85 & 191 & 340 & 531 & 765 & 1041 & 1360 & 1721 & 2125 \\
\hline 90 & 203 & 360 & 563 & 810 & 1103 & 1446 & 1823 & 2250 \\
\hline
\end{tabular}

${ }^{\mathrm{a}} \mathrm{SMI}$ was calculated using the formula: $\mathrm{SMI}=(\text { grade })^{2} \times \%$ motile (Bavister, 1974). ${ }^{\mathrm{b}}$ The initial motility grade of sperm suspensions used in this study was 3.5 . The minimum percentage motile sperms was $60 \%$.

mentation to gamete-co-culture medium on fertilization rate and the pronuclear event after fertilization. Mature mouse oocytes $(n=461)$ were co-cultured with sperms in TYH with ETO (treatments). Other mature oocytes ( $n=437)$ were co-culture with sperm in ETO free TYH (controls). The co-culture was allowed to continue for $5 \mathrm{~h}$. The treatments and controls were further cultured for $24 \mathrm{~h}$ in KSOM with ETO and KSOM without ETO, respectively. Pronuclear formation was assed as described above.

\section{Statistical analysis}

The date of sperm motility in experiment I were subjected to 2-way analysis of variance (ANOVA) and presented as mean \pm SE. Data of fertilized oocytes were evaluated with Chi-square followed by Fisher's exact test. Differences at a probability of $p<0.05$ were considered significant.

\section{RESULTS}

\section{Experiment I}

The results showed no interaction $(p>0.05)$ between time of incubation and ETO supplementation to culture media. ETO supplementation to culture media had no effect on sperm motility. The grade of sperm motility increased gradually by time to reach grade 5 after $2 \mathrm{~h}$. Therefore, this mean that SMI increased gradually to reach their peak values after $2 \mathrm{~h}$ of incubation (Figure 1ad). The mean SMI after 30 min was $873.0 \pm 52.3$, after 1 $h$ was $1140.0 \pm 68.3$, after $1.5 \mathrm{~h}$ was $1443.0 \pm 86.5$, after $2 \mathrm{~h}$ was $1781.3 \pm 106.7$, after $2.5 \mathrm{~h}$ was $1781.3 \pm 106.7$, and after $3 \mathrm{~h}$ was $1781.3 \pm 106.7$. These values are equal for both treatments and control. Most of the sperms in the sperm suspensions become hyperactivated (capacitized) after $2 \mathrm{~h}$ of incubation. No significant ( $p>0.05$ ) differences were observed among the SMI of treatments and controls.

\section{Experiment II}

As shown in Table 2, the percentage (85\%) of oocytes fertilized in the treatments $(n=412)$ was significantly $(p<$ $0.05)$ lower than the percentage $(93.3 \%)$ of oocytes fertilized in the controls $(n=408)$. None of the oocytes fertilized in the treatments formed male or female pronuclei, while $92.6 \%$ of the oocytes fertilized in the controls $(n=378)$ formed pronuclei. All the oocytes fertilized in treatments $(100 \%)$ had enlarged or decondensed sperm heads, while only 30 oocytes $(7.4 \%)$ of the controls had enlarged or decondensed sperm heads (Figure 2 ).

\section{DISSCUSION}

The results of the present study showed that suspension of mouse epididymal spermatozoa in a medium supplemented with ETO has no effect on sperm motility and/or capacitation. Furthermore, co-culture of mouse oocytes with sperms in a fertilization medium supplemented with ETO did not impair fertilization. When the sperms penetrated the oocytes, their heads enlarged but further decondensation to form male pronuclei was completely inhibited. 

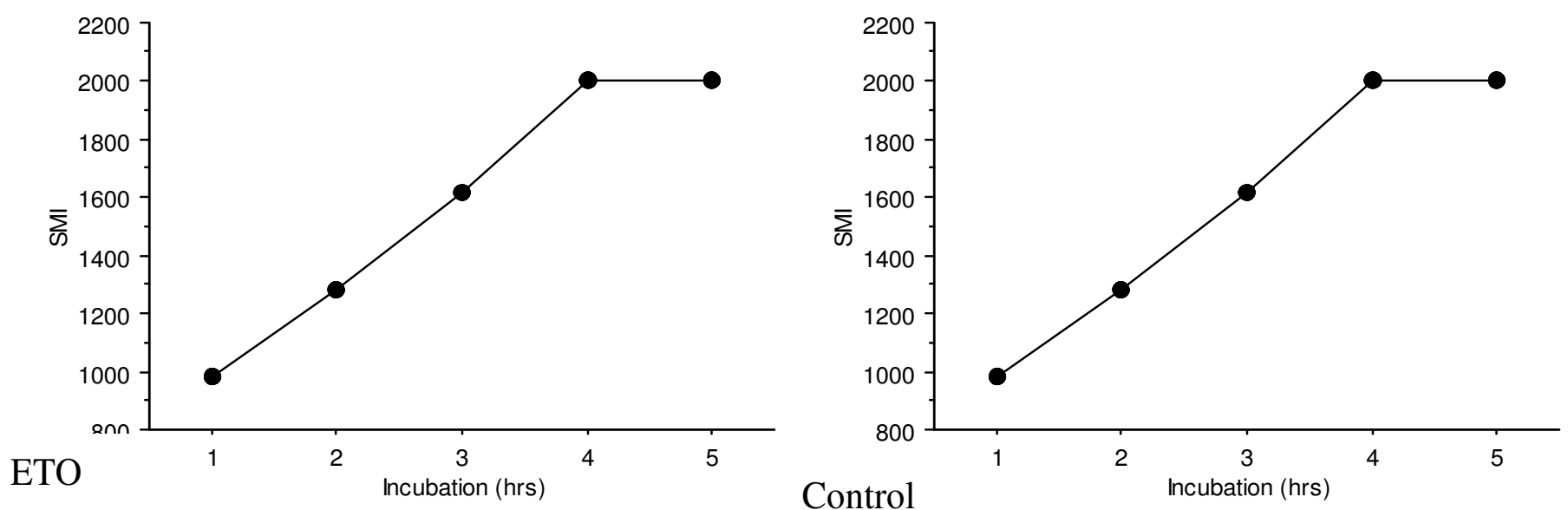

Figure 1a. Comparison between SMI of the sperm suspensions of the male mice in A treatments (ETO) and control. Data are presented as means \pm SE. The SMI increased gradually in the treatments and the controls without no differences $(p>0.05)$ among them.
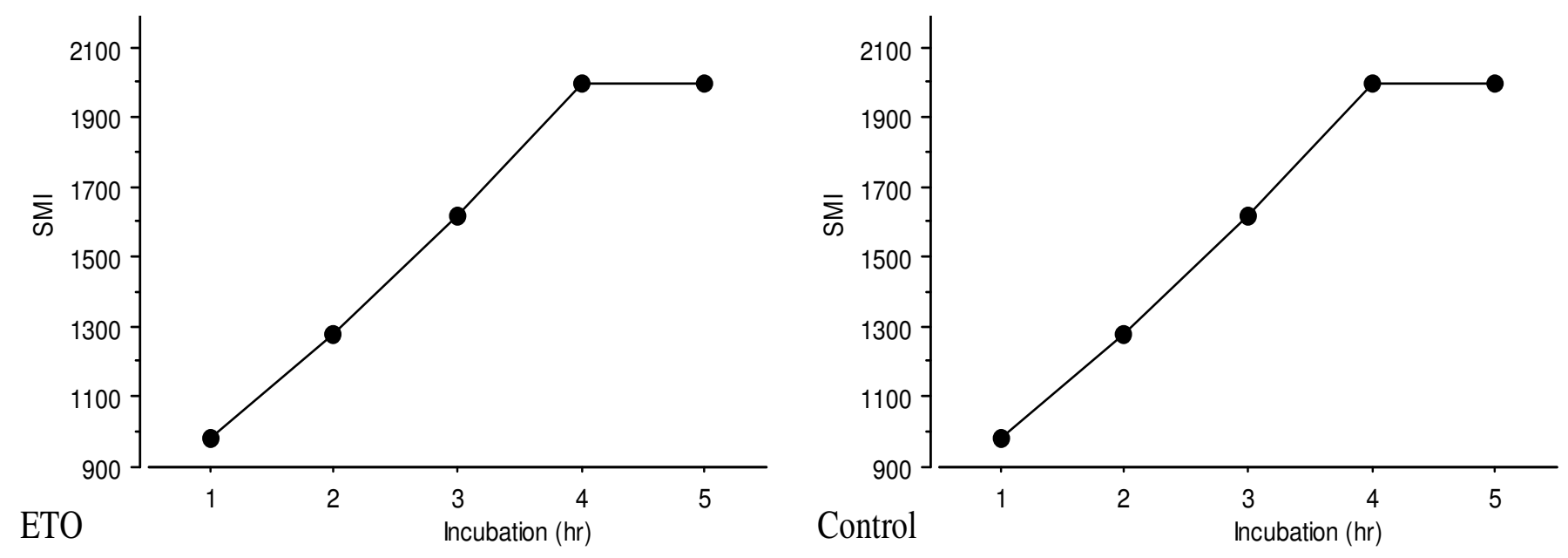

Figure 1b. Comparison between SMI of the sperm suspensions of the male mice in B treatment (ETO) and control. Data are presented as means $\pm S E$. The SMl increased gradually in the treatments and the controls without no differences $(p>0.05)$ among them.
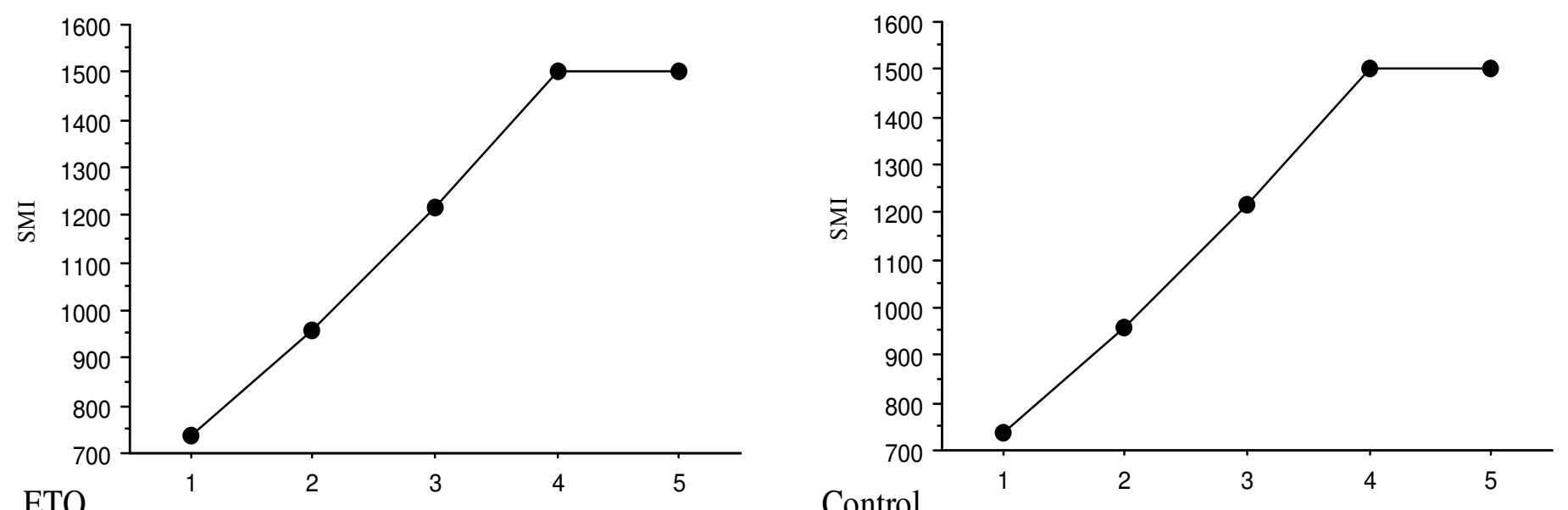

Figure 1c. Comparison between SMI of the sperm suspensions of the male mice in C treatments (ETO) and control. Data are presented as means \pm SE. The SMI increased gradually in the treatments and the controls without no differences $(p>0.05)$ among them. 

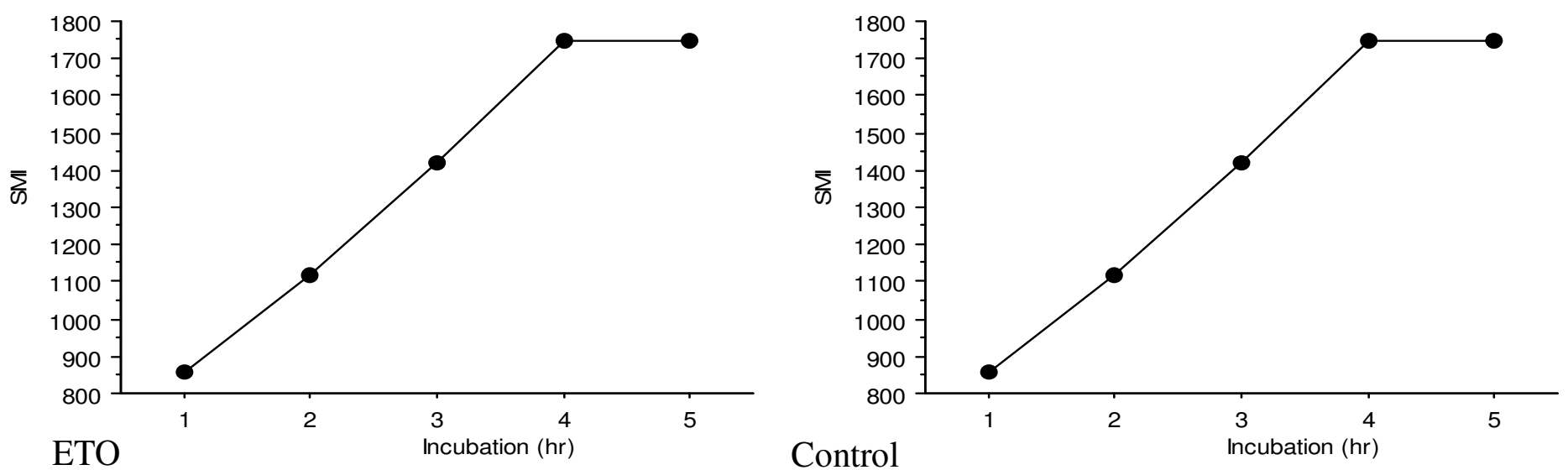

Figure 1d. Comparison between SMI of the sperm suspensions of the male mice in D treatment (ETO) and control. Data are presented as means \pm SE. The SMI increased gradually in the treatments and the controls without no differences $(p>0.05)$ among them.

Table 2. Nuclear events that happened in mouse oocytes fertilized in treatments and controls ${ }^{\mathrm{a}}$.

\begin{tabular}{lcccc}
\hline \multirow{2}{*}{$\begin{array}{c}\text { Treatment } \\
\text { groups }\end{array}$} & $\begin{array}{c}\text { No. of oocytes } \\
\text { examined }\end{array}$ & $\begin{array}{c}\text { No. (\%) of oocytes } \\
\text { Fertilized }\end{array}$ & Pronuclei & $\begin{array}{c}\text { Enlarged or decondensed } \\
\text { sperm heads }\end{array}$ \\
\hline Treatments & 461 & $412(85.0)^{\star}$ & $0(0)^{\star \star}$ & $412(100)^{\star \star}$ \\
Controls & 437 & $408(93.3)$ & $378(92.6)$ & $30(7.4)$ \\
\hline
\end{tabular}

${ }^{\mathrm{a}}$ Treatments are sperm suspension and fertilization drops containing $50 \mu \mathrm{g} / \mathrm{ml}$ ETO whereas controls are ETO free sperm suspension and fertilization drops. ${ }^{*} * *$ Significantly different from the controls $(p<b 0.05, p<0.001$, respectively).
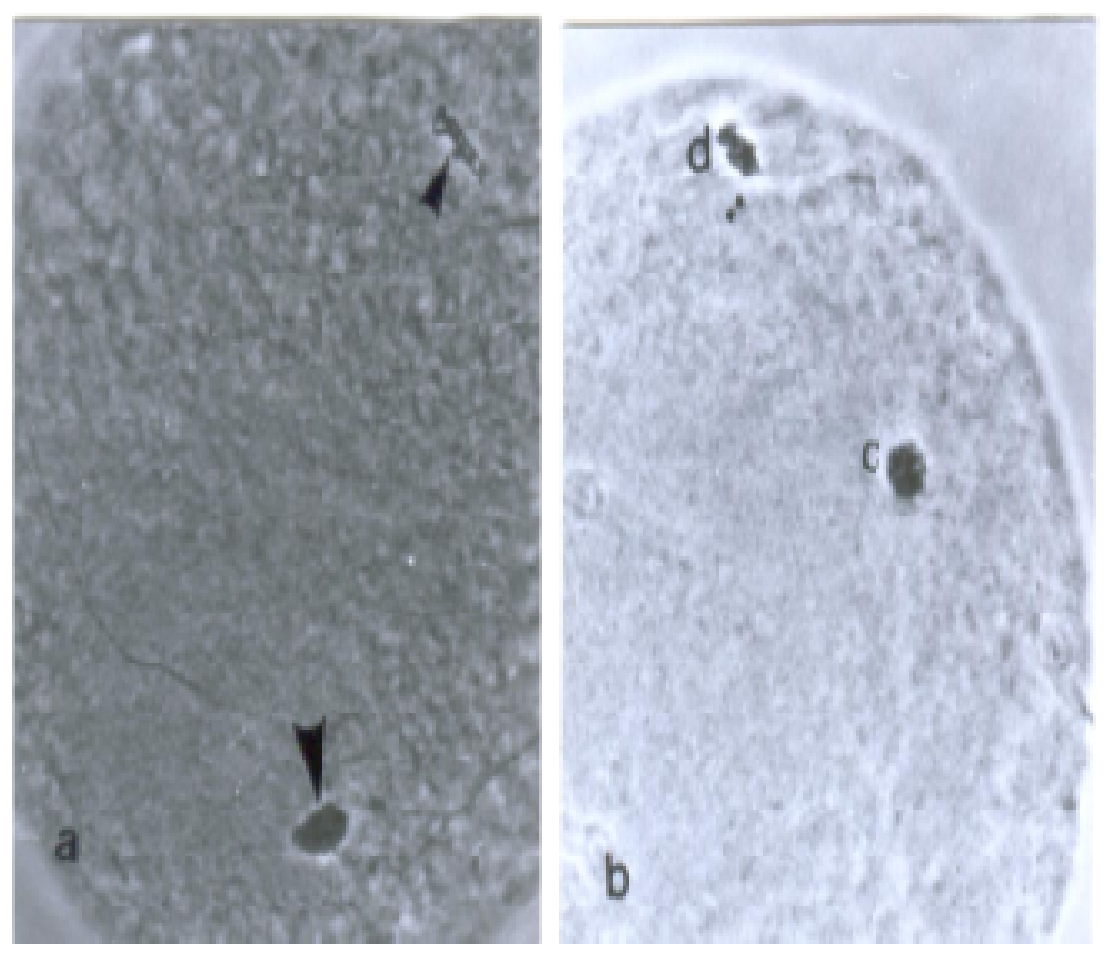

Figure 2. Oocytes fertilized in culture medium supplemented with ETO. Oocyte (a) with sperm head (large arrow head) and metaphase II spindle (small arrow head). Oocyte (b) with decondensed sperm head (c) and metaphase II spindle (d). 
Mouse spermatozoa suspended in TYH medium become capacitated after $2 \mathrm{~h}$ of incubation (Toyoda et al., 1971). The capacitation is associated with hyperactivation (Yanagimachi, 1970). Hyperactivation is a type of motility given grade 5 by Bavister (1974) and Bavister and Andrews (1988). In the present study when mouse spermatozoa were suspended in ETO supplemented TYH medium, they became hyperactivated after $2 \mathrm{~h}$ of incubation. ETO is a chemical known to block top 2 enzyme (Fulka and Moor, 1993; Gaudio and Risely, 1994; Elsheikh et al., 1997; Elsheikh et al., 2004). Furthermore, ETO has adverse effect on cumulus cells (Elsheikh et al., 2004). ETO supplementation to the culture medium did not influence sperm motility and capacitation.

High percentages of oocytes in the current study were fertilized in the presence of ETO. However, the percentage of oocytes fertilized in ETO free medium was higher. Since the sperm capacitation was not influenced by ETO, the reduction in the fertilization percentage was attributed to the influence of ETO on the cumulus cells surrounding the oocytes (Elsheikh et al., 2004). Although, the culture duration in this study was enough for the fertilized oocytes to form pronuclei, none of the oocytes fertilized in ETO supplemented media failed to form neither male pronuclei nor female pronuclei. Top 2 is essential for decondensation of chromosome (Dinardo et al., 1994; Wright and Schatten, 1990; Downes et al., 1991). Thus top 2 is necessary to aid sperm head decondensation. When ETO was used in the fertilization media decondensation of sperm heads that penetrated the oocytes was impaired. This is probably due to blocking of top 2 enzymes. This finding confirms previous finding (Elsheikh et al., 2004).

It is concluded that, ETO has no effect on sperm motility, capacitation and/or sperm fertilizing ability. Furthermore, top 2 activities in mouse sperm heads appears after oocyte penetration.

\section{REFERENCES}

Bavister BD, Andrews VC (1988). A rapid sperm motility bioassay procedure for quality control testing of water and culture media. J. In Vitro Fert. Emb. Trans. 5(2): 67-75.
Bavister BP (1974). The effect of variations in culture conditions on motility of hamaster spermatozoa. J. Reprod. Fert. 38: 431-440.

Chen JL, Longo FJ (1986). Expression and Localization of DNA topoisomerase 2 during rat spermatogenesis. Mol. Reprod. Dev. 45: 61-71.

Dinardo S, Voelkel K, Sternglanz R (1984). DNA topoisomerase 2 mutant of Saccharomyces cerevisiae: topoisomerase 2 is required for segregation of daughter molecules at termination of DNA replication. Proc. Nat. Acad. Sci. USA, 81: 2616-2620.

Downes CS, Mullinger AM, Johnson RT (1991). Inhibitors of DNA topoisomerase 2 prevent chromatid separation in mammalian cells but do not prevent exit from mitosis. Proc. Natl. Acad. Sci. USA, 88: 8895-8899.

Earnshow WC, Heck MS (1985a). Localization of topoisomerase 2 in mitotic chromosomes. J. Cell Biol. 100: 1716-1725.

Earnshow WC, Halligan B, Cooke CA, Heck MS, Liu LF (1985b). Topoisomerase 2 is a structural component of mitotic chromosome scaffolds. J. Cell Biol. 100: 1706-1715.

Elsheikh AS, Takahashi Y, Hishinuma M, Kanagawa H (1997). Developmental ability of mouse late 2-cell stage blastomeres fused to chemically enucleated oocytes in vitro. Jpn. J. Vet. Med. Sci. 59: 107113.

Elsheikh AS, Takahashi Y, Kanagawa H (2004). Possible involvement of DNA topoisomeras 2 enzyme in the process of meiotic arrest in mouse metaphase 2 oocytes. J. Anim. Vet. Adv. 3(11): 776-780.

Erbach GT, Lawitts JA, Papaioannou VE, Biggers JD (1994). Differential growth of mouse preimplantation embryos in chemically defined media. Biol. Reprod. 50: 1027-1033.

Fulka Jr. J, Moor RM (1993). Noninvasive chemical enucleation of mouse oocytes. Mol. Reprod. Dev. 34: 427-430.

Gasser SM, Laroche T, Falquet J, Laemmli UK (1986). Metaphase chromosome structure involvement of topoisomerase 2. J. Mol. Biol. 188: 613-629.

Gaudio MM, Risley MS (1994). Topoisomerase 2 expression and VM26 induction of DNA breaks during spermatogenesis in Xenopus laevis. J. Cell Sci. 107: 2887-2898.

Toyoda Y, Chang MC (1974). Fertilization of rat's eggs in vitro by epididymal spermatozoa and the development of eggs following transfer. J. Reprod. Fertil. 36: 9-22.

Toyoda Y, Yokoyama M, Hoshi T (1971). Studies on the fertilization of mouse eggs in vitro. I. In vitro fertilization of eggs by fresh epididymal sperms. Jpn. J. Anim. Reprod. 16: 147-151.

Wright S, Schatten G (1990). Teniposide, a topoisomerase 2 inhibitor, prevents chromosome condensation and separation but not decondensation in fertilized Surf Clam (Spisula solidissima) oocytes. Dev. Biol. 14: 224-232.

Yanagimachi R (1970). The movement of golden hamster spermatozoa before and after capacitation. J. Reprod. Fert. 23: 477-480. 